\title{
Exploring the neural mechanisms of finasteride: A proteomic analysis in the nucleus accumbens
}

\author{
Alessio Soggiu ${ }^{\mathrm{a}}$, Cristian Piras ${ }^{\mathrm{a}}$, Viviana Greco ${ }^{\mathrm{b}}$, Paola Devoto ${ }^{\mathrm{c}}$, Andrea Urbani ${ }^{\mathrm{b}, \mathrm{d}}$, Luigino Calzetta ${ }^{\mathrm{e}}$, \\ Marco Bortolato ${ }^{\mathrm{f}}$, Paola Roncada, $\mathrm{PhD}^{\mathrm{g}}{ }^{\text {* }}$ \\ a Dipartimento di Medicina Veterinaria, Università degli Studi di Milano, Italy \\ ${ }^{\mathrm{b}}$ Fondazione S.Lucia-IRCSS, Roma, Italy \\ ${ }^{c}$ Department of Biomedical Sciences, Section of Neuroscience and Clinical Pharmacology, University of Cagliari, Monserrato, CA, Italy \\ ${ }^{\mathrm{d}}$ Istituto di Biochimica e Biochimica Clinica, Università Cattolica del Sacro Cuore, Roma, Italy \\ e Department of Systems Medicine, University of Rome Tor Vergata, Chair of Respiratory Medicine, Rome, Italy \\ ${ }^{\mathrm{f}}$ Department of Pharmacology and Toxicology, School of Pharmacy, University of Utah, Salt Lake City, UT, USA \\ g Istituto Sperimentale Italiano "Lazzaro Spallanzani", Milano, Italy
}

\section{A R T I C L E IN F O}

\section{Article history:}

Received 3 May 2016

Received in revised form 7 September 2016

Accepted 5 October 2016

Available online $\mathrm{xxx}$

Keywords:

Rat brain

Finasteride

Proteomics

Gene ontology

\section{A B S T R A C T}

The enzyme $5 \alpha$-reductase $(5 \alpha \mathrm{R})$ catalyzes the conversion of progesterone and testosterone into neuroactive steroids implicated in a wide array of behavioral functions. The prototypical $5 \alpha \mathrm{R}$ inhibitor, finasteride (FIN), is clinically approved for the treatment of androgenic alopecia and benign prostatic hyperplasia. Recent evidence has shown that FIN, albeit generally well tolerated, can induce untoward psychological effects in a subset of patients; furthermore, this drug may have therapeutic efficacy for a number of different neuropsychiatric conditions, ranging from Tourette syndrome to schizophrenia. In rat models of these conditions, FIN has been shown to block the effects of dopamine receptors in the nucleus accumbens (NAcc), a key terminal of the dopamine mesolimbic system. The biological underpinnings of these effects, however, remain mostly elusive. To elucidate the neurochemical networks that may be responsible for the behavioral effects of FIN, we evaluated the proteomic profile of the NAcc following acute $(100 \mathrm{mg} / \mathrm{kg}, \mathrm{IP})$ and subchronic (7 days; $100 \mathrm{mg} / \mathrm{kg} / \mathrm{day}, \mathrm{IP})$ treatment with this drug, in comparison with vehicle treatment $(\mathrm{n}=5 / \mathrm{group})$. Two-dimensional electrophoresis (2-DE) analysis coupled to mass spectrometry revealed significant changes in the expression of nine proteins (CRMP2, PSMD1, STX18, KCNC3, CYP255, GABRP, GABT, PRPS1, CYP2B3), which were further analyzed by ontological classification (PANTHER). These results point to a number of novel potential chemical targets of FIN, and may help elucidate the underpinnings of FIN's behavioral effects and therapeutic potential for neuropsychiatric disorders.

(C) 2016 Published by Elsevier Ltd.

\section{Introduction}

The enzyme $5 \alpha$-reductase $(5 \alpha \mathrm{R})$ catalyzes the rate-limiting step in the irreversible saturation of the 4,5 double bond of the A ring of $\Delta^{4}-3$ ketosteroids, such as progesterone and testosterone (Paba et al., 2011). The products of this enzymatic process include a number of neuroactive steroids, including 5 $\alpha$-dihydroprogesterone (DHP) and $5 \alpha$-dihydrotestosterone (DHT); in the brain, these steroids are further converted into key neurosteroid metabolites implicated in behavioral regulation, such as $3 \alpha, 5 \alpha$, tetrahydroprogesterone (allopregnanolone, AP) and $3 \alpha, 5 \alpha$,androstanediol ( $3 \alpha-$ diol) (Barbaccia, 2004; Frye et al., 2010). The prototypical $5 \alpha \mathrm{R}$ inhibitor, finasteride (FIN), is currently approved for the treatment of a number of conditions associated with high DHT, such as benign prostatic hyperplasia (BPH) and androge-

* Corresponding author at: Istituto Sperimentale Italiano "Lazzaro Spallanzani" Milano, Sezione di Proteomica c/o Dipartimento di Medicina Veterinaria, Università degli Studi di Milano, via Celoria 10, 20133 Milano, Italy.

Email address: paola.roncada@istitutospallanzani.it, paola.roncada@gmail.com (P. Roncada) netic alopecia. Although FIN use is typically well-tolerated, its use for either BPH or alopecia has been linked to disturbances in ejaculation and erection, as well as loss of libido (Carbone and Hodges, 2003; Kaufman et al., 1998; McClellan and Markham, 1999). Clinical trials have shown that these side effects affect $\sim 15 \%$ patients, in comparison with $7 \%$ of placebo-treated counterparts (Traish et al., 2011). Recent lines of evidence have also shown that FIN can induce enduring psychoendocrinological side effects in vulnerable patients, including depression and reduction of libido (Irwig, 2012; Rahimi-Ardabili et al., 2006; Traish et al., 2015). This symptomatology can persist after therapy cessation, a condition that has been recently termed "post-finasteride syndrome" (PFS) and may be underpinned by persistent changes in the neurosteroid profile in CSF and plasma (Caruso et al., 2015).

Complementary to this concept, FIN may have therapeutic potential for several neuropsychiatric conditions characterized by information-processing deficits and poor behavioral control, encompassing Tourette syndrome to schizophrenia and problem gambling in Parkinson's disease patients (Bortolato et al., 2012, 2007; Koethe et al., 2008; Paba et al., 2011). In parallel with these observations, FIN was shown to ameliorate several behavioral abnormalities exhibited by rat 
models of the conditions shown by Bortolato and coworkers (Bortolato et al., 2008; Frau et al., 2015, 2016), likely by reducing the effects of dopamine signaling at the level of $\mathrm{D}_{1}$, and possibly $\mathrm{D}_{3}$, receptors (Frau et al., 2016). Consistently with these findings, the antipsychotic-like actions of FIN have been shown to be primarily contributed by the nucleus accumbens (NAcc) (Devoto et al., 2012), the major terminal of the dopamine mesolimbic pathway. These mechanisms may also account for the lowering of mood and libido, given the importance of the dopaminergic signaling in the NAcc in the regulation of sex drive, reward, and motivation (Giuliano and Allard, 2001; Ikemoto and Panksepp, 1999). While these premises point to FIN as a promising option in neuropsychiatric therapy, the specific mechanisms whereby this drug may reduce dopaminergic signaling remain elusive, partially reflecting our limited knowledge of the molecular targets of neurosteroids in the brain. Based on this background, the present study was aimed at the investigation of the proteomic profile of the NAcc of rats treated with either acute or subchronic FIN treatment. Specifically, the intraperitoneal dose of FIN that was administered was $100 \mathrm{mg} / \mathrm{kg}$, which was previously shown to induce a robust reduction of allopregnanolone levels, increase concentrations of pregnenolone and progesterone (Frau et al., 2015), and counter the effects of dopaminergic agonists across multiple behavioral paradigms (Bortolato et al., 2008; Devoto et al., 2012).

\section{Materials and methods}

\subsection{Animals and treatment}

A total of 15 Sprague-Dawley male rats (Harlan Italy, S. Pietro al Natisone, Italy), weighing 200-250 g were used for these experiments. Animals were group-housed in cages $(\mathrm{n}=3$ /cage) with ad libitum access to food and water. Rooms were maintained at $22 \pm 0.2{ }^{\circ} \mathrm{C}$ on reversed 12-h light/dark cycle (with lights off at 07:00 PM). All experimental procedures were in compliance with the National Institutes of Health guide for the care and use of Laboratory animals and approved by the local Institutional Animal Use Committee. This study was carried out in agreement with ARRIVE guidelines.

\subsection{Drugs and treatments}

FIN (Sigma-Aldrich, Milan, Italy) was suspended in a vehicle (VEH) solution containing 5\% Tween 80 and 95\% saline, and administered intraperitoneally (IP) at the dose of $100 \mathrm{mg} / \mathrm{kg}$ (in an injection volume of $2 \mathrm{ml} / \mathrm{kg}$ body weight). In every cage, each rat was subjected to one of the following three treatments: 1) 7-day administration of VEH $(n=5) ; 2) 6$-day administration of VEH, followed by FIN on the seventh day $(\mathrm{n}=5) ; 3) 7$-day administration of FIN $(\mathrm{n}=5)$. All efforts were made to minimize animal suffering. One hour following the last treatment, rats were sacrificed and their NAcc was harvested, rapidly frozen in dry ice, and stored at $-80{ }^{\circ} \mathrm{C}$ until analysis.

\subsection{Protein extraction and 2-dimensional electrophoresis (2-DE)}

NAcc samples were harvested according to the indication of the atlas of Paxinos and Watson (1998). Proteins were extracted from all NAcc tissue samples using a variant of the protocol described in (Roncada et al., 2009). Briefly, tissues were weighed and suspended $(1: 10, \mathrm{w} / \mathrm{v})$ in a solution of $7 \mathrm{M}$ urea, $2 \mathrm{M}$ thiourea, $2 \% 3$-[(3-Cholamidopropyl)dimethylammonio-1-propanesulfonate (CHAPS), $2 \%$ Triton $\mathrm{X}-100,2 \%(\mathrm{v} / \mathrm{v})$ Ampholine $\mathrm{pH}$ 3.5-10. The suspensions were subjected to five cycles of bead beating (Biospec Products Inc, USA) for $1 \mathrm{~min}$ at $4000 \mathrm{rpm} / \mathrm{min}$ and finally centrifuged at 14 $000 \mathrm{~g}$ and $4{ }^{\circ} \mathrm{C}$ for $30 \mathrm{~min}$ to remove cellular debris and insoluble materials and the supernatants were collected. Protein concentration on supernatants was determined using the 2D Quant Kit (GE Healthcare, Little Chalfont, UK). Seven-cm immobilized $\mathrm{pH}$ gradient (IPG) strips with a linear $\mathrm{pH}$ range of 4-7 (GE Healthcare) were rehydrated overnight in $125 \mu \mathrm{l}$ of buffer containing $7 \mathrm{M}$ urea, $2 \mathrm{M}$ thiourea $2 \%$ CHAPS, $1 \%$ DTT, $2 \%$ ampholine $\mathrm{pH} 3.5-10.100 \mu \mathrm{g}$ of protein sample were loaded on each IPG strip using cup loading at the cathodic side (Piras et al., 2012). Isoelectric focusing was performed using an Ettan IPGphor III IEF system (GE Healthcare) at $20^{\circ} \mathrm{C}$ with a current of $50 \mu \mathrm{A}$ per strip. For IEF the following protocol was used: $30 \mathrm{~V}$ (4 h), $50 \mathrm{~V}(3 \mathrm{~h}), 100 \mathrm{~V}(3 \mathrm{~h}), 500 \mathrm{~V}(3 \mathrm{~h}), 1000 \mathrm{~V}(3 \mathrm{~h}), 3000 \mathrm{~V}(3 \mathrm{~h})$, $4000 \mathrm{~V}(3 \mathrm{~h}), 6000 \mathrm{~V}(3 \mathrm{~h})$ and $8000 \mathrm{~V}(8 \mathrm{~h})$ (Soggiu et al., 2013). After the first dimension, IPG strips were equilibrated twice with a solution containing $6 \mathrm{M}$ urea, 2\% SDS, $50 \mathrm{mM}$ Tris- $\mathrm{HCl} \mathrm{pH} 8.8$ and 30\% glycerol, for $15 \mathrm{~min}$, under gentle stirring. For the first equilibration step was used $1 \%$ DTT and for the second $2.5 \%$ iodoacetamide. The second dimension was performed using homemade $12 \%$ acrylamide vertical SDS-PAGE slab gels on a Mini Protean Tetra cell (BioRad, Hercules, CA). IPG strips were put on top of the SDS gels and were poured up to $1 \mathrm{~cm}$ from the top of the plates and then sealed with $1 \mathrm{ml}$ of a solution containing $0.5 \%$ low-melting-point agarose diluted in hot SDS running buffer (25 mM Tris- $\mathrm{HCl} \mathrm{pH}$ 8.3, $192 \mathrm{mM}$ glycine, 0.1\% SDS). Molecular weight protein markers (Invitrogen, Carlsbad, CA) were applied on one end of the IPG strips. In the second dimension, gels were run until the bromophenol blue front-line came out of the gel. After runs, gels were stained with colloidal Coomassie and digitized with ImageScanner III (GE Healthcare) at 600 dpi.

\subsection{Image analysis and spots identification}

Gel images were imported into Progenesis SameSpots (v4.6; Totallab Ltd, Newcastle, UK) for analysis. All imported images were processed to check image quality (saturation, dimension, background). The aligned images were then automatically analyzed using the $2 \mathrm{D}$ analysis module for spot detection, background subtraction, normalization, and spot matching, and all spots were manually reviewed and validated to ensure proper detection and matching. Differentially expressed spots with a $p$ value $<0.05$ and a power value $>0.8$ were selected for the identification by mass spectrometry.

\subsection{Protein identification by MALDI-TOF/TOF MS analysis}

Protein identification was performed according to protocols described by (Piras et al., 2015). Briefly, single spots were excised from gels and gradually dehydrated. After reduction and alkylation, single spots were digested with a solution of $0.01 \mu \mathrm{g} / \mu \mathrm{l}$ of porcine trypsin (Promega, Madison, WI) at $37{ }^{\circ} \mathrm{C}$ overnight. (Arioli et al., 2009). The reaction was stopped with $1 \%$ TFA in $\mathrm{H}_{2} \mathrm{O}$ (v/v). Peptides were desalted and concentrated by $\mathrm{C} 18$ ZipTip (Millipore) and co-crystallized with a solution of $0.5 \mathrm{mg} / \mathrm{ml} \alpha$-ciano-4-hydroxycinnamic acid dissolved in $50 \%$ acetonitrile, $0.05 \%$ TFA on a Ground Steel plate (Bruker-Daltonics, Bremen, Germany) previously spotted with a thin layer of $10 \mathrm{mg} / \mathrm{ml} \alpha$-ciano-4-hydroxycinnamic acid dissolved in Ethanol/Acetonitrile/0.1\% (v/v) TFA in $\mathrm{H}_{2} \mathrm{O}$. MS raw data were acquired with an Ultraflex III MALDI-TOF/TOF spectrometer (Bruker Daltonics) in positive reflectron mode. External calibration was performed using the standard peptide mixture calibration $(\mathrm{m} / \mathrm{z}$ : 
1046.5418, 1296.6848, 1347.7354, 1619.8223, 2093.0862, 2465.1983, 3147.4710; Bruker-Daltonics). FlexAnalysis 3.3 software (Bruker-Daltonics) was used for the selection of the monoisotopic peptide masses of each mass spectra. Internal calibration was performed on autolysis peaks of porcine trypsin $(\mathrm{m} / \mathrm{z}: 842.509$ and 2211.104). After exclusion of contaminant ions (known matrix and human keratin peaks), the created peak lists were analyzed by MASCOT v.2.4.1 algorithm (www.matrixscience.com) against SwissProt_2013_05 database restricted to Rattus norvegicus taxonomy (7867 sequences). The query for Peptide Mass Fingerprinting (PMF) analysis was performed with carbamidomethylation of cysteines as fixed modification, oxidation of methionines as variable modification, one missed cleavage site allowed for trypsin and $70 \mathrm{ppm}$ as maximal tolerance. Mascot protein scores greater than 52 were considered significant $(\mathrm{p}<0.05)$ for protein identification assignment. To confirm PMF analysis, the instrument was switched to LIFT mode and subsequently MS/MS spectra were acquired (Piras et al., 2015). For the fragmentation, precursor ions were manually selected and the precursor mass window was automatically set.

The analyses of each MS/MS spectra (spectra baseline subtraction, smoothing Savitsky-Golay and centroiding) were carried out by Flex Analysis 3.3 software (Bruker Daltonics). These parameters used for database search were: carbamidomethylation of cysteines and oxidation on methionine respectively for fixed and variable modifications, one missed cleavage, $50 \mathrm{ppm}$ for the mass tolerance for precursor ions and 0.4 Da for fragments. The taxonomy was restricted to Rattus norvegicus. The confidence interval for protein identification was set to $95 \%(\mathrm{p}<0.05)$ and only peptides with Individual ions scores $>22$, that indicate identity or extensive homology, were considered.

\subsection{Bioinformatics analysis}

To identify Gene Ontology (GO) classes and biologically relevant molecular pathways from our data, proteomics datasets were analyzed using the PANTHER (Protein ANalysis THrough Evolutionary Relationships) Classification System (version 10, Released 2015-05-15) (Mi et al., 2016). In particular, the PANTHER statistical overrepresentation Test (release 20160321) was applied to retrieve statistically significant ( $\mathrm{p}$-value $<0.05$ ) over-represented or enriched functional classes and pathways.

\subsection{Statistical analyses}

All comparisons between treatment groups were calculated on PRISM 5 (GraphPad software, USA) applying one-way ANOVA, followed by Tukey's test for post hoc comparisons. A p-value of 0.05 was considered statistically significant.

\section{Results}

All proteins were separated by 2-DE and identified by MALDI-TOF/TOF mass spectrometry (Fig. 1). As shown in Table 1 , this analysis revealed that either acute or subchronic treatment with FIN elicited significant changes in the expression of nine proteins in the NAcc (Fig. 2). Specifically, this analysis detected that acute FIN administration led to the increased expression of the collapsin response mediator protein 2 (CRMP2), Syntaxin-18 (STX18), the non-ATPase regulatory subunit of the 26S proteasome (PSMD1) as well as Cytochrome P450 2B3 (CYP2B3) ( $<<0.05$ for all comparisons with VEH-treated controls). In contrast, the same treatment led to the decrease of GABA transaminase (GABT) $(\mathrm{P}<0.05)$.

The 7-day FIN treatment led to the decreased expression of the voltage-gated potassium channel KCNC3, Cytochrome P450 2C55

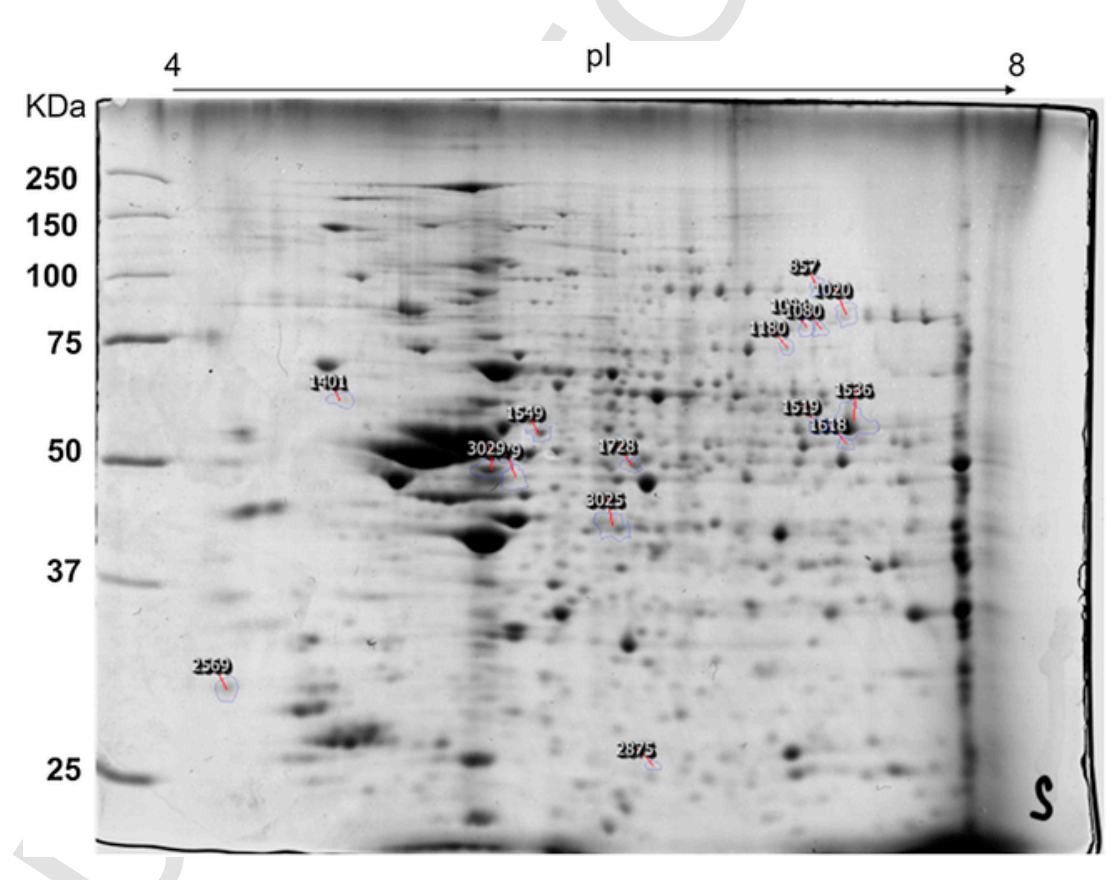

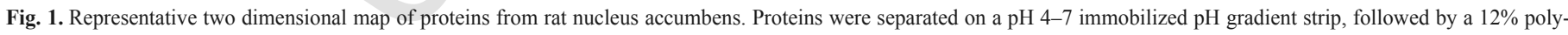

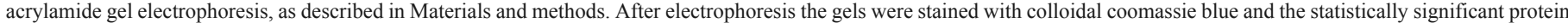

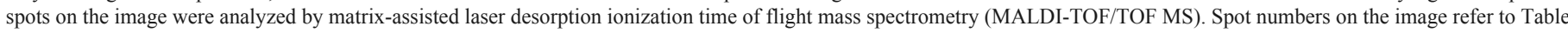
1. (For interpretation of the references to colour in this figure legend, the reader is referred to the web version of this article.) 
Table 1

Protein identification by MALDI-TOF/TOF MS analysis.

\begin{tabular}{|c|c|c|c|c|c|c|c|c|c|c|}
\hline \multirow[t]{2}{*}{$\begin{array}{l}\text { Spot } \\
\text { no. }\end{array}$} & \multirow[t]{2}{*}{$\underline{\text { Protein description }}^{\mathrm{a}}$} & \multirow[t]{2}{*}{$\underline{\text { Accession no. }^{b}}$} & \multirow[t]{2}{*}{$\begin{array}{l}\text { Fold } \\
\text { change }\end{array}$} & \multirow[t]{2}{*}{$\frac{\text { Theoretical }}{\mathrm{M}_{r}(\mathrm{kDa}) / \mathrm{pI}}$} & \multicolumn{3}{|l|}{$\underline{\mathrm{PMF}}$} & \multicolumn{3}{|l|}{$\underline{\mathrm{MS} / \mathrm{MS}}$} \\
\hline & & & & & $\underline{\text { Mascot }} \underline{\underline{\text { scorec }}}$ & $\frac{\% \text { Seq. }}{\text { coverage }^{\mathrm{d}}}$ & $\frac{\text { Matched }}{\text { peptides }^{\mathrm{e}}}$ & $\underline{\text { Peptide sequence }}^{\mathrm{f}}$ & $\frac{\text { Mascot }}{\text { score }^{\mathrm{g}}}$ & $\underline{\mathrm{m} / \mathrm{z}^{\mathrm{h}}}$ \\
\hline 1549 & $\begin{array}{l}\text { Dihydropyrimidinase-related } \\
\text { protein } 2\end{array}$ & DPYL2_RAT & 1.4 & $62.6 / 5.95$ & 106 & 26 & $13 / 49$ & ${ }^{472}$-KPFPDFVYK- ${ }^{480}$ & 80 & 1140.62 \\
\hline 1084 & $\begin{array}{l}\text { Potassium voltage-gated } \\
\text { channel subfamily } \mathrm{C} \text { member } \\
3\end{array}$ & KCNC3_RAT & 2 & $95.6 / 6.62$ & 125 & 27 & $19 / 71$ & $\begin{array}{l}452 \text {-IVLEDGTLHVTEGSGR-- } \\
317 \text {-IPLDVGHRGAGNSTTTAK- }{ }^{334}\end{array}$ & $\begin{array}{l}80 \\
92\end{array}$ & $\begin{array}{l}1682.87 \\
1794.83\end{array}$ \\
\hline 1519 & Cytochrome P450 2C55 & CP255_RAT & 1.5 & $56.6 / 6.51$ & 214 & 77 & $31 / 126$ & ${ }^{453}$-NCIGKQFAMNELK- ${ }^{465}$ & 80 & 1553.89 \\
\hline 825 & $\begin{array}{l}\text { 26S proteasome non-ATPase } \\
\text { regulatory subunit } 1\end{array}$ & PSMD1_RAT & 1.8 & $106.7 / 5.25$ & 168 & 23 & $25 / 59$ & ${ }^{153}$-SATLVLAYLMIHK- ${ }^{165}$ & 86 & 1475.78 \\
\hline 1748 & $\begin{array}{l}\text { Gamma-aminobutyric acid } \\
\text { receptor subunit pi }\end{array}$ & GBRP_RAT & 1.8 & $50.8 / 8.03$ & 116 & 45 & $14 / 67$ & ${ }^{92}$-WTDPRLVFEGNK- ${ }^{103}$ & 76 & 1461.80 \\
\hline 1785 & $\begin{array}{l}\text { 4-aminobutyrate } \\
\text { aminotransferase, } \\
\text { mitochondrial }\end{array}$ & GABT_RAT & 1.9 & $57.2 / 8.15$ & 186 & 42 & $21 / 59$ & ${ }^{174}$-TIFMWYR_- ${ }^{180}$ & 80 & 1016.50 \\
\hline 2394 & $\begin{array}{l}\text { Ribose-phosphate } \\
\text { pyrophosphokinase } 1\end{array}$ & PRPS1_RAT & 1.4 & $35.3 / 6.51$ & 160 & 47 & $13 / 29$ & $\begin{array}{l}{ }^{368} \text {-EEFRPSAPYR- }{ }^{377} \\
{ }^{85} \text {-VTAVIPCFPYAR- }{ }^{96}\end{array}$ & $\begin{array}{l}110 \\
80\end{array}$ & $\begin{array}{l}1251.61 \\
1394.71\end{array}$ \\
\hline 3025 & Syntaxin-18 & STX18_RAT & 1.4 & $38.7 / 5.56$ & 180 & 52 & $22 / 77$ & ${ }^{265}$-LQEIFTEK- ${ }^{272}$ & 70 & 1007.55 \\
\hline 3028 & Cytochrome P450 2B3 & CP2B3_RAT & 1.2 & $56.86 / 6.39$ & 117 & 37 & $14 / 49$ & ${ }^{158}$-IMADSVNIMLDK- ${ }^{169}$ & 70 & 1365.64 \\
\hline
\end{tabular}

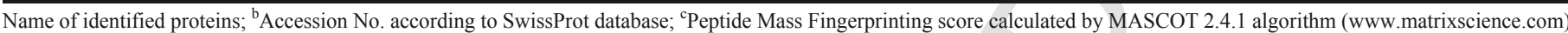

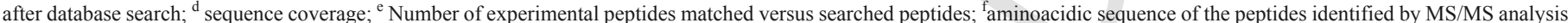

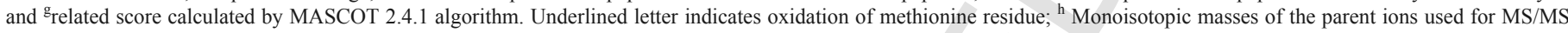
analysis.

CYP2B3

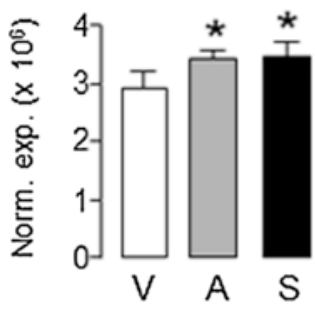

CRMP2

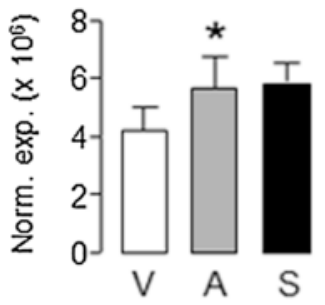

GABT

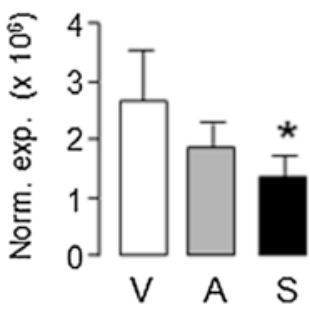

STX18

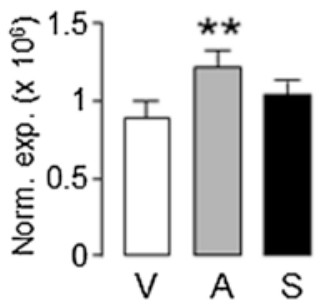

KCNC3

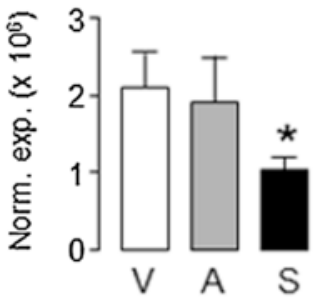

GABRP

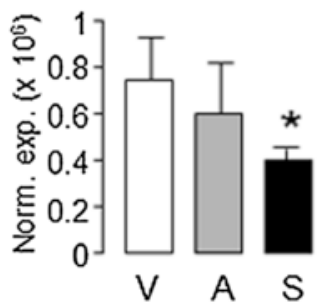

PRPS1

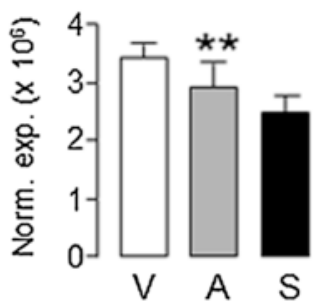

PSMD1

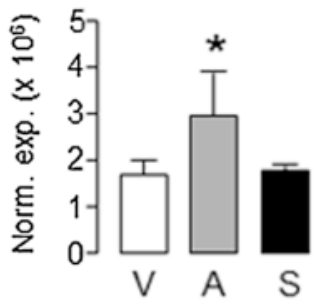

CYP255

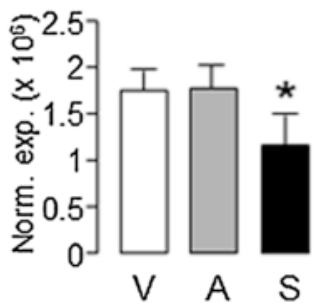

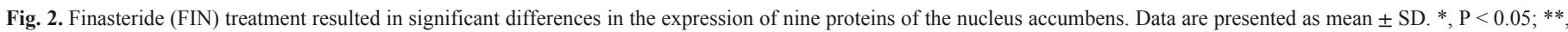
$\mathrm{P}<0.01$ in comparison with vehicle-treated rats; V, vehicle; A, acute (i.e., 6 days of vehicle followed by one day of FIN); S, sub-chronic (7 days of FIN). 
(CYP255), the $\pi$ subunit of the GABA-A receptor (GBRP), phosphoribosyl pyrophosphate synthetase-1 (PRPS1) and GABT (Ps $<0.05)$, while it significantly increased the levels of CYP2B3 $(\mathrm{P}<0.05)$.

As shown in Table 2, gene ontology analyses by PANTHER revealed that the proteins affected by FIN treatment belong to key functional pathways underlying signaling processes in the central nervous system (synaptic transmission, exocytosis, ion transport), or were included in regulatory pathways for neurosteroid synthesis (steroid and fatty acid conversion).

Among the pathways and molecular functions indicated by PANTHER, a number of processes appear to be relevant to the potential role of FIN in behavioral regulation and dopamine signaling modulation, such as the synthesis and catabolism of GABA, as well as the axonal structure and degradation ubiquitin-proteasome dependent and the regulation of the activity of GABA-A receptor and voltage-gated ion channels (Tables 3 and 4).

\section{Discussion}

The results of this study showed that the administration of an acute, high dose of FIN (100 mg/kg, IP) associated with marked behavioral effects (Bortolato et al., 2008; Frau et al., 2015) led to a significant increase in the expression of four proteins (CRMP2, STX18, PSMD1, CYP2B3), as well as the reduction of GABT levels, in the NAcc; conversely, subchronic FIN treatment led to the decrease of five proteins (KCNC3, CYP255, PRPS1, GBRP, and GABT) and a significant up-regulation of CYP2B3. Gene ontology analyses, as highlighted by PANTHER, indicated that FIN treatment results in over-representation of six different pathways compared to the control, including GABA synthesis and degradation, as well as pyrimidine metabolism. These alterations were associated with multiple key biological processes, including synaptic transmission and fatty acid metabolism, as well as the regulation of the steroid biosynthetic process.

Table 2

PANTHER overrepresented pathways related to the finasteride treatment.

\begin{tabular}{|c|c|c|c|c|c|}
\hline PANTHER Pathways & Ref & $\begin{array}{l}\text { Our } \\
\text { list }\end{array}$ & Expected & $\begin{array}{l}\text { Fold } \\
\text { Enrichment }\end{array}$ & $\begin{array}{c}\mathrm{P} \\
+/-\quad \text { value }\end{array}$ \\
\hline Pyrimidine Metabolism & 12 & 2 & 0.00 & $>100$ & $* * *$ \\
\hline Aminobutyrate degradation & 4 & 1 & 0.00 & $>100$ & $* *$ \\
\hline $\begin{array}{l}\text { Gamma-aminobutyric acid } \\
\text { synthesis }\end{array}$ & 5 & 1 & 0.00 & $>100$ & $* *$ \\
\hline $\begin{array}{l}\text { Axon guidance mediated by } \\
\text { semaphorins }\end{array}$ & 22 & 1 & 0.01 & $>100$ & $* *$ \\
\hline De novo purine biosynthesis & 41 & 1 & 0.02 & 61.40 & $* *$ \\
\hline $\begin{array}{l}\text { Ubiquitin proteasome } \\
\text { pathway }\end{array}$ & 66 & 1 & 0.03 & 38.14 & + \\
\hline Unclassified & 19872 & 5 & 7.89 & 0.63 & - \\
\hline
\end{tabular}

The first column contains the name of the annotation data category. The second column (REF) contains the number of genes in the Rattus norvegicus gene reference list that map to each particular annotation data category in the first column. The third column (our list) contains the number of genes in our dataset that map to the annotation data category. The fourth column contains the expected value, which is the number of genes that we expect in our list for each category, based on the reference list. The fifth column shows the Fold Enrichment of the genes observed in the analyzed protein list over the expected (all the proteins in our list divided by the expected number). If it is greater than one, it indicates that the category is overrepresented in our protein list. Conversely, the category is under-represented if it is less than one. The sixth column has either a + or - A plus sign indicates over-representation of related category in our experiment. Conversely, a negative sign indicates under-representation. The seventh column is the $\mathrm{p}$-value as determined by the binomial statistic. A p-value $<0.05$ indicates a statistically significant value. $\mathrm{p}$ value legend: $* \mathrm{P}<0.05 ; * * \mathrm{P}<0.01 ; * * * \mathrm{P}<0.001$. Potentially relevant processes related to FIN treatment are evidenced in bold.
Table 3

PANTHER biological process overrepresentation test, see Table 2 for column details.

\begin{tabular}{|c|c|c|c|c|c|c|}
\hline GO biological process complete & Ref & $\begin{array}{l}\text { Our } \\
\text { list }\end{array}$ & Expected & $\begin{array}{l}\text { Fold } \\
\text { Enrichment }\end{array}$ & $+/-$ & $\begin{array}{l}\mathrm{P} \\
\text { value }\end{array}$ \\
\hline epoxygenase $\mathrm{P} 450$ pathway & 25 & 2 & 0.01 & $>100$ & + & $* * *$ \\
\hline $\begin{array}{l}\text { arachidonic acid metabolic } \\
\text { process }\end{array}$ & 55 & 2 & 0.02 & 91.54 & + & $* * *$ \\
\hline $\begin{array}{l}\text { positive regulation of aspartate } \\
\text { secretion }\end{array}$ & 1 & 1 & 0 & $>100$ & + & $* * *$ \\
\hline regulation of aspartate secretion & 1 & 1 & 0 & $>100$ & + & $* * *$ \\
\hline $\begin{array}{l}\text { long-chain fatty acid metabolic } \\
\text { process }\end{array}$ & 87 & 2 & 0.03 & 57.87 & + & $* * *$ \\
\hline Eicosanoids metabolic process & 96 & 2 & 0.04 & 52.44 & + & $* * *$ \\
\hline $\begin{array}{l}\text { fatty acid derivative metabolic } \\
\text { process }\end{array}$ & 96 & 2 & 0.04 & 52.44 & + & $* * *$ \\
\hline $\begin{array}{l}\text { unsaturated fatty acid metabolic } \\
\text { process }\end{array}$ & 104 & 2 & 0.04 & 48.41 & + & $* * *$ \\
\hline $\begin{array}{l}\text { gamma-aminobutyric acid } \\
\text { catabolic process }\end{array}$ & 2 & 1 & 0 & $>100$ & + & $* * *$ \\
\hline $\begin{array}{l}\text { 5-phosphoribose } 1 \text {-diphosphate } \\
\text { biosynthetic process }\end{array}$ & 3 & 1 & 0 & $>100$ & + & $* *$ \\
\hline $\begin{array}{l}\text { 5-phosphoribose } 1 \text {-diphosphate } \\
\text { metabolic process }\end{array}$ & 3 & 1 & 0 & $>100$ & + & $* *$ \\
\hline $\begin{array}{l}\text { gamma-aminobutyric acid } \\
\text { biosynthetic process }\end{array}$ & 3 & 1 & 0 & $>100$ & + & $* *$ \\
\hline response to drug & 620 & 3 & 0.25 & 12.18 & + & $* *$ \\
\hline $\begin{array}{l}\text { negative regulation of gamma- } \\
\text { aminobutyric acid secretion }\end{array}$ & 4 & 1 & 0 & $>100$ & + & $* *$ \\
\hline positive regulation of ER to & 4 & 1 & 0 & $>100$ & + & $* *$ \\
\hline $\begin{array}{l}\text { gamma-aminobutyric acid } \\
\text { metabolic process }\end{array}$ & 5 & 1 & 0 & $>100$ & + & $* *$ \\
\hline $\begin{array}{l}\text { regulation of neurotransmitter } \\
\text { levels }\end{array}$ & 171 & 2 & 0.07 & 29.44 & + & $* *$ \\
\hline $\begin{array}{l}\text { negative regulation of } \\
\text { dopamine secretion }\end{array}$ & 6 & 1 & 0 & $>100$ & + & $* *$ \\
\hline $\begin{array}{l}\text { positive regulation of dopamine } \\
\text { metabolic process }\end{array}$ & 6 & 1 & 0 & $>100$ & + & $* *$ \\
\hline $\begin{array}{l}\text { positive regulation of } \\
\text { catecholamine metabolic } \\
\text { process }\end{array}$ & 6 & 1 & 0 & $>100$ & + & $* *$ \\
\hline $\begin{array}{l}\text { positive regulation of inhibitory } \\
\text { postsynaptic potential }\end{array}$ & 7 & 1 & 0 & $>100$ & + & $* *$ \\
\hline $\begin{array}{l}\text { modulation of inhibitory } \\
\text { postsynaptic potential }\end{array}$ & 8 & 1 & 0 & $>100$ & + & $* *$ \\
\hline AMP biosynthetic process & 8 & 1 & 0 & $>100$ & + & $* *$ \\
\hline $\begin{array}{l}\text { neurotransmitter catabolic } \\
\text { process }\end{array}$ & 10 & 1 & 0 & $>100$ & + & $* *$ \\
\hline drug catabolic process & 12 & 1 & 0 & $>100$ & + & $* *$ \\
\hline $\begin{array}{l}\text { positive regulation of glutamate } \\
\text { secretion }\end{array}$ & 12 & 1 & 0 & $>100$ & + & $* *$ \\
\hline $\begin{array}{l}\text { regulation of ER to Golgi } \\
\text { vesicle-mediated transport }\end{array}$ & 13 & 1 & 0.01 & $>100$ & + & $* *$ \\
\hline fatty acid metabolic process & 300 & 2 & 0.12 & 16.78 & + & $* *$ \\
\hline $\begin{array}{l}\text { positive regulation of cellular } \\
\text { amine metabolic process }\end{array}$ & 15 & 1 & 0.01 & $>100$ & + & $* *$ \\
\hline $\begin{array}{l}\text { negative regulation of } \\
\text { catecholamine secretion }\end{array}$ & 16 & 1 & 0.01 & $>100$ & + & $* *$ \\
\hline $\begin{array}{l}\text { regulation of dopamine } \\
\text { metabolic process }\end{array}$ & 19 & 1 & 0.01 & $>100$ & + & $* *$ \\
\hline $\begin{array}{l}\text { regulation of catecholamine } \\
\text { metabolic process }\end{array}$ & 20 & 1 & 0.01 & $>100$ & + & $* *$ \\
\hline $\begin{array}{l}\text { modulation of synaptic } \\
\text { transmission }\end{array}$ & 357 & 2 & 0.14 & 14.1 & + & $* *$ \\
\hline $\begin{array}{l}\text { regulation of glutamate } \\
\text { secretion }\end{array}$ & 23 & 1 & 0.01 & $>100$ & + & $* *$ \\
\hline $\begin{array}{l}\text { regulation of dopamine } \\
\text { secretion }\end{array}$ & 25 & 1 & 0.01 & $>100$ & + & $* *$ \\
\hline $\begin{array}{l}\text { neurotransmitter metabolic } \\
\text { process }\end{array}$ & 25 & 1 & 0.01 & $>100$ & + & $* *$ \\
\hline drug metabolic process & 32 & 1 & 0.01 & 78.67 & + & $*$ \\
\hline $\begin{array}{l}\text { regulation of cellular amine } \\
\text { metabolic process }\end{array}$ & 42 & 1 & 0.02 & 59.94 & + & $*$ \\
\hline $\begin{array}{l}\text { regulation of catecholamine } \\
\text { secretion }\end{array}$ & 50 & 1 & 0.02 & 50.35 & + & $*$ \\
\hline $\begin{array}{l}\text { regulation of neurotransmitter } \\
\text { secretion }\end{array}$ & 59 & 1 & 0.02 & 42.67 & + & $*$ \\
\hline
\end{tabular}


Table 3 (Continued)

\begin{tabular}{|c|c|c|c|c|c|c|}
\hline GO biological process complete & Ref & $\begin{array}{l}\text { Our } \\
\text { list }\end{array}$ & Expected & $\begin{array}{l}\text { Fold } \\
\text { Enrichment }\end{array}$ & $+/-$ & $\begin{array}{l}P \\
\text { value }\end{array}$ \\
\hline $\begin{array}{l}\text { regulation of neurotransmitter } \\
\text { transport }\end{array}$ & 70 & 1 & 0.03 & 35.96 & + & $*$ \\
\hline synaptic vesicle transport & 85 & 1 & 0.03 & 29.62 & + & $*$ \\
\hline $\begin{array}{l}\text { establishment of synaptic vesicle } \\
\text { localization }\end{array}$ & 85 & 1 & 0.03 & 29.62 & + & $*$ \\
\hline synaptic vesicle localization & 87 & 1 & 0.03 & 28.93 & + & * \\
\hline
\end{tabular}

$\mathrm{p}$ value legend: $* \mathrm{P}<0.05 ; * * \mathrm{P}<0.01 ; * * * \mathrm{P}<0.001$

Table 4

PANTHER molecular function overrepresentation test.

\begin{tabular}{|c|c|c|c|c|c|c|}
\hline GO molecular function complete & Ref & $\begin{array}{l}\text { Our } \\
\text { list }\end{array}$ & Expected & $\begin{array}{l}\text { Fold } \\
\text { Enrichment }\end{array}$ & $+/-$ & $\begin{array}{l}\mathrm{P} \\
\text { value }\end{array}$ \\
\hline $\begin{array}{l}\text { arachidonic acid epoxygenase } \\
\text { activity }\end{array}$ & 33 & 2 & 0.01 & $>100$ & + & $* * *$ \\
\hline $\begin{array}{l}\text { arachidonic acid monooxygenase } \\
\text { activity }\end{array}$ & 35 & 2 & 0.01 & $>100$ & + & $* * *$ \\
\hline aromatase activity & 38 & 2 & 0.02 & $>100$ & + & $* * *$ \\
\hline steroid hydroxylase activity & 55 & 2 & 0.02 & 91.54 & + & $* * *$ \\
\hline $\begin{array}{l}\text { succinate-semialdehyde } \\
\text { dehydrogenase binding }\end{array}$ & 1 & 1 & 0 & $>100$ & + & $* * *$ \\
\hline $\begin{array}{l}\text { 4-aminobutyrate transaminase } \\
\text { activity }\end{array}$ & 1 & 1 & 0 & $>100$ & + & $* * *$ \\
\hline $\begin{array}{l}\text { (S)-3-amino-2-methylpropionate } \\
\text { transaminase activity }\end{array}$ & 1 & 1 & 0 & $>100$ & + & $* * *$ \\
\hline $\begin{array}{l}\text { extracellular-glutamate-gated } \\
\text { chloride channel activity }\end{array}$ & 2 & 1 & 0 & $>100$ & + & $* * *$ \\
\hline monooxygenase activity & 132 & 2 & 0.05 & 38.14 & + & $* *$ \\
\hline $\begin{array}{l}\text { ribose phosphate } \\
\text { diphosphokinase activity }\end{array}$ & 5 & 1 & 0 & $>100$ & + & $* *$ \\
\hline diphosphotransferase activity & 8 & 1 & 0 & $>100$ & + & $* *$ \\
\hline AMP binding & 12 & 1 & 0 & $>100$ & + & $* *$ \\
\hline gated channel activity & 304 & 2 & 0.12 & 16.56 & + & $* *$ \\
\hline GABA-A receptor activity & 19 & 1 & 0.01 & $>100$ & + & $* *$ \\
\hline $\begin{array}{l}\text { extracellular-glutamate-gated } \\
\text { ion channel activity }\end{array}$ & 20 & 1 & 0.01 & $>100$ & + & $* *$ \\
\hline GABA receptor activity & 22 & 1 & 0.01 & $>100$ & + & $* *$ \\
\hline $\begin{array}{l}\text { transferase activity, transferring } \\
\text { nitrogenous groups }\end{array}$ & 30 & 1 & 0.01 & 83.91 & + & $*$ \\
\hline $\begin{array}{l}\text { passive transmembrane } \\
\text { transporter activity }\end{array}$ & 439 & 2 & 0.17 & 11.47 & + & * \\
\hline SNAP receptor activity & 36 & 1 & 0.01 & 69.93 & + & $*$ \\
\hline ADP binding & 40 & 1 & 0.02 & 62.93 & + & $*$ \\
\hline $\begin{array}{l}\text { excitatory extracellular ligand- } \\
\text { gated ion channel activity }\end{array}$ & 51 & 1 & 0.02 & 49.36 & + & $*$ \\
\hline GDP binding & 60 & 1 & 0.02 & 41.96 & + & $*$ \\
\hline $\begin{array}{l}\text { extracellular ligand-gated ion } \\
\text { channel activity }\end{array}$ & 71 & 1 & 0.03 & 35.46 & 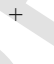 & $*$ \\
\hline chloride channel activity & 77 & 1 & 0.03 & 32.69 & + & $*$ \\
\hline anion channel activity & 87 & 1 & 0.03 & 28.93 & + & $*$ \\
\hline $\begin{array}{l}\text { chloride transmembrane } \\
\text { transporter activity }\end{array}$ & 88 & 1 & 0.03 & 28.61 & + & $*$ \\
\hline $\begin{array}{l}\text { voltage-gated potassium } \\
\text { channel activity }\end{array}$ & 89 & 1 & 0.04 & 28.28 & + & $*$ \\
\hline $\begin{array}{l}\text { ion transmembrane transporter } \\
\text { activity }\end{array}$ & 828 & 2 & 0.33 & 6.08 & + & $*$ \\
\hline $\begin{array}{l}\text { inorganic anion transmembrane } \\
\text { transporter activity }\end{array}$ & 112 & 1 & 0.04 & 22.48 & + & $*$ \\
\hline potassium channel activity & 120 & 1 & 0.05 & 20.98 & + & $*$ \\
\hline
\end{tabular}

$\mathrm{p}$ value legend: $* \mathrm{P}<0.05 ; * * \mathrm{P}<0.01 ; * * * \mathrm{P}<0.001$

As mentioned above, the FIN dose used in this study was shown to acutely reduce allopregnanolone levels and increase concentrations of pregnenolone and progesterone in the striatum of rats (Frau et al., 2015). Allopregnanolone and other $5 \alpha \mathrm{R}$ substrates and/or products play an important role in the functional regulation of GABA-A receptors and are posited to modulate mitochondrial and bioenergetic functions (Klinge, 2008; Papadopoulos et al., 2006; Robertson et al., 2006). Taken together, these results reveal that FIN may induce a dis- crete number of neurochemical alterations in the proteome of the NAcc, which may be related to its antidopaminergic properties (Frau et al., 2016). In the following sections, we will analyze how each of the 9 proteins affected by FIN treatment may be relevant to the behavioral and neurochemical effects of this drug.

\subsection{Proteins up-regulated by FIN treatment}

The only protein that was up-regulated by both acute and subchronic FIN treatment was the enzyme CYP2B3, a rat-specific isoform of the CYP2B family (Desrochers et al., 1996) homologous to CYP2B6 in humans. The increase in CYP2B3 after FIN treatment, irrespective of its duration, suggests a robust interaction between $5 \alpha$ Rs and this member of the cytochrome P450 family. Like other members of this family, CYP2B3 may be upregulated to enable the metabolism of either FIN itself or a number of steroid precursors that no longer undergo conversion into their $5 \alpha$-reduced metabolites. Accordingly, the CYP2B family has been shown to catalyze testosterone 16-beta hydroxylation (Ekins et al., 1998), thereby activating an alternative degradation pathway for this ketosteroid. If the upregulation of CYP2B3 is indeed important in limiting potentially detrimental effects of FIN (or possibly mediating some of its therapeutic effects), it may be interesting to verify whether the effects of FIN may be modified by changes in CYP2B expression/activity. Of note, CYP2B6 is implicated in the metabolism of several psychotropic drugs, such as the antidepressant bupropion (Hesse et al., 2000), and variants of its gene have been shown to exhibit alterations in the effects of this drug (Zhu et al., 2012).

Our data showed that three additional proteins were down-regulated by acute, but not subchronic FIN treatment:

- PSMD1, an essential component of the regulatory particle of the $26 \mathrm{~S}$ proteasome that recognizes and guides target proteins towards the proteolytic site (da Fonseca and Morris, 2008). Given the crucial role of the proteasome system in protein degradation, the FIN-induced reduction in PSMD1 levels are predicted to result in the reduced catabolism of critical target proteins in the NAcc. In this respect, it is worth noting that the $26 \mathrm{~S}$ proteasome is implicated in the modulation of the receptors and behavioral effects of progesterone (Camacho-Arroyo et al., 2002; González-Flores et al., 2004).

- STX18, a member of the syntaxin family largely involved in the progression of vesicles from the endoplasmic reticulum and the cis-Golgi compartment (Hatsuzawa et al., 2000; Nakajima et al., 2004). Given that both $5 \alpha \mathrm{R} 1$ and $5 \alpha \mathrm{R} 2$ are located in the endoplasmic reticulum, these data suggest that their acute inhibition may trigger a number of changes in membrane transport across intracellular compartment and vesicles;

- CRMP2, a member of the collapsin response mediator proteins that regulates cytoskeletal dynamics and vesicle trafficking by binding to tubulin heterodimers and promoting microtubule assembly (Fukata et al., 2002; Hensley et al., 2011); this protein also modulates the function and expression of $\mathrm{N}$-type calcium channels (Brittain et al., 2011), which facilitate neurotransmitter release from the presynaptic terminals (Ronzitti et al., 2014), and serve as downstream effectors of $\mathrm{D}_{2}$-like dopamine autoreceptors (Cabrera-Vera et al., 2004; Canti and Dolphin, 2003; Ramanathan et al., 2008). Taken together, this evidence suggests that the FIN-induced increase in CRMP2 expression in the NAcc may lead to changes in dopamine release from mesolimbic projections. In line with this idea, we recently showed that acute administration of the same doses of FIN used in this study $(100 \mathrm{mg} / \mathrm{kg}$, IP) produces a significant increase in the accumbal dopamine release in rats (Frau et al., 
2016). The mechanisms whereby acute FIN treatment increases CRMP2 may reflect the action of neuroactive steroids on the multiple targets of this protein. For example, interactions between pregnenolone and microtubules or testosterone and N-type calcium channels (Nudler et al., 2005) have been described in the literature. CRMP2 has also been reported to interact functionally with NMDA glutamate receptors (Brustovetsky et al., 2014), pivotal controllers of the integration of afferent input in medium spiny neurons of the NAcc (Wolf et al., 2005). Notably, multiple neurosteroids have been shown to act as allosteric modulators of NMDA receptors (Borovska et al., 2012; Elfverson et al., 2008; Johansson et al., 2010, 2008; Sedlacek et al., 2008; Vyklicky et al., 2015). Irrespective of the specific mechanism, it is worth noting that CRMP2 is implicated in multiple neuropathological processes in several neuropsychiatric disorders (Hensley et al., 2011), highlighting the possibility that CRMP2 may act as a key effector for the behavioral effects of FIN.

\subsection{Proteins down-regulated by FIN treatment}

The only protein that was down-regulated by both acute and subchronic FIN treatment was GABT. This mitochondrial enzyme is responsible for the main catabolic process of GABA, and its reaction is based on the transfer of the amino group from GABA to alpha-ketoglutarate, with consequent production of L-glutamate and succinic semialdehyde (Sherif, 1994; Tillakaratne et al., 1995). The rapid conversion of this metabolite into succinic acid (which enters Krebs cycle) ensures that the reaction catalyzed by GABA-T is physiologically unidirectional. The FIN-induced decrease in GABA-T in the NAcc is predicted to increase GABA concentrations and reduce glutamate synthesis, thereby favoring an inhibitory tone in the brain. Given that FIN leads to the reduction of the synthesis of AP, a positive allosteric modulator of GABA-A receptor and negative allosteric modulator of NMDA receptors, it is likely that the changes in GABA-T may be enacted by the brain as a compensatory process to offset the imbalance in excitation/inhibition induced by FIN.

Four additional proteins were down-regulated by subchronic, but not acute, FIN treatment:

- GBRP, the $\pi$ subunit of GABA-A receptor, which was originally characterized in the uterus (Hedblom and Kirkness, 1997), and has been found to reduce the sensitivity of this channel to allopregnanolone (Neelands and Macdonald, 1999). Although GBRP expression has been attested in the brain (Allen Brain Atlas; http: //mouse.brain-map.org/gene/show/85019), very little is currently known about its role in the CNS. Our finding of a reduction in this subunit following FIN subchronic treatment opens up to the possibility that this subunit may also be instrumental in increasing the sensitivity of GABA-A to allopregnanolone and, possibly, other neuroactive steroids, possibly as a compensatory phenomenon for the prolonged suppression of allopregnanolone induced by subchronic $5 \alpha \mathrm{R}$ inhibition;

- KCNC3, the potassium channels that mediate currents (Kv3.3) characterized by a marked rightward shift in their voltage dependence as well as a very fast rate of deactivation upon repolarization (Coetzee et al., 1999; Rudy et al., 1999). Due to these characteristics, the inactivation of $\mathrm{KCNC} 3$ is highly variable, and may be modified by numerous physiological conditions (Fernandez et al., 2003; Rae and Shepard, 2000; Rudy et al., 1999). These unique biophysical properties may account for the bimodal behavior of the membrane of medium spiny neurons in the NAcc, which oscillates between a hyperpolarized state dominated by an inwardly rectify- ing potassium current (Uchimura et al., 1989) and a more depolarized state dominated by rapidly inactivating currents (Stern et al., 1997; Wilson and Kawaguchi, 1996), such as those mediated by $\mathrm{KCNC} 3$. Although little is known about the regulation of KCNC3 by steroids, our data point to the possibility that prolonged suppression of neurosteroidogenesis may lead to changes in potassium currents and electric properties of accumbal cells. In keeping with this hypothesis, preliminary evidence has pointed to a role of neurosteroids and sex hormones in the regulation of voltage-gated $\mathrm{K}^{+}$ channels (Drici et al., 1996; Wang et al., 1998; Wong et al., 2008).

- PRPS1, a key enzyme for the production of phosphoribosyl pyrophosphate (PRPP), an essential cofactor for the de novo biosynthesis of purines, pyrimidines and their nucleotides, including ATP and GTP (Kornberg et al., 1954; Lieberman et al., 1955; Zimmer, 1998). Notably, recent evidence has shown that multiple neurosteroids exert beneficial bioenergetic effects on the mitochondrion and its ability to produce ATP (Grimm et al., 2014). From this perspective, our finding suggests that $5 \alpha \mathrm{R}$ products may have a trophic effect on these processes by increasing the synthesis of PRPS1. In line with this evidence, it is also worth noting that allopregnanolone acts as a positive allosteric modulator of ATP-gated channels (Codocedo et al., 2009), potentially suggesting a sophisticated relationship between neurosteroids and ATP-mediated actions.

- CYP2C55, a poorly-characterized member of the CYP2C family; although, to the best of our knowledge, no information is currently available on the role of CYP2C55 in the brain, or in the metabolism of neuroactive steroids, our findings point to the possibility that this target may be implicated in brain functions, in line with preliminary evidence on other CYP2C members, such as CYP2C13 (Riedl et al., 2000) and 2C9 (Gervasini et al., 2001).

\subsection{Limitations and conclusions}

Several limitations of the present study ought to be acknowledged. First, our analysis could not distinguish the specific effects of FIN in the two major subdivisions of the NAcc, the core and the shell. These two areas serve distinct roles in the regulation of reward-seeking behaviors. For example, dopamine neurotransmission in the shell is instrumental for the appraisal of reward and encoding of motivational salience for reinforcing stimuli; conversely, the core is implicated in the modulation of instrumental conditioning and delayed reinforcement (Ambroggi et al., 2011; Cardinal and Cheung, 2005; Corbit et al., 2001; Di Chiara, 2002). Further studies will be required to assess the specific roles of neurosteroids and $5 \alpha$ Rs in these two regions. Second, our animal models were based on acute and subchronic administration of a high FIN dose; while these preparations are useful to assess the potential implications of the NAcc proteome in the behavioral effects of FIN, they bear only limited face, construct and predictive validity with respect to the conditions secondary to FIN treatment in humans; furthermore, the relevance of our findings to the underpinnings of PFS is limited, given that the present study did not include the assessment of enduring effects of FIN after its discontinuation. Finally, our studies were only limited to the NAcc; however, other regions, such as the prefrontal cortex, may be involved in the behavioral effects of FIN (Devoto et al., 2012). These limitations notwithstanding, our results showed that FIN treatment affected the expression of a number of accumbal proteins involved in key functional processes, such as regulation of GABAergic neurotransmission, as well as steroid and pyrimidine metabolism. These findings may prove crucial to understanding the neurochemical mechanisms of FIN's neuropsychiatric side effects, as well as its po- 
tential therapeutic properties for neurological and mental disorders (Paba et al., 2011).

\section{Conflict of interest}

All authors declare that they have no conflicts of interests.

\section{Funding sources}

This research was partially supported by grants from the National Institute of Mental Health (NIH R01 MH104603, to MB), Tourette Syndrome Association (to MB), as well as a sub-award from the NIH grant P20 GM103638 (to MB), and a grant from the Regional Government of Sardinia (to PD and AS). None of the institutions had any further role in study design; in the collection, analysis and interpretation of data; in the writing of the report; and in the decision to submit the paper for publication.

\section{Author contributions}

All authors have given approval to the final version of the manuscript.

Alessio Soggiu, performed most of the proteomics experiments, bioinformatic analysisand wrote the manuscript.

Cristian Piras: provided helpful inputs on data analysis and contributed to write the manuscript

Viviana Greco, performed most of the mass spectrometry analysis.

Andrea Urbani supervised mass spectrometry experiments and contributed to write the manuscript.

Luigi Calzetta revised manuscript and contributed in data interpretation.

Paola Devoto and Marco Bortolato designed the experimental study, contributed to write the manuscript and to interprete experimental data.

Paola Roncada supervised the project and contributed to edit the manuscript.

\section{Acknowledgments}

The authors wish to thank Drs. Roberto Frau and Valentina Bini for their technical support.

\section{References}

Ambroggi, F., Ghazizadeh, A., Nicola, S.M., Fields, H.L., 2011. Roles of nucleus accumbens core and shell in incentive-cue responding and behavioral inhibition. J. Neurosci. 31, 6820-6830.

Arioli, S., Roncada, P., Salzano, A.M., Deriu, F., Corona, S., Gusglielmetti, S., Bonizzi, L., Scaloni, A., Mora, D., 2009. The relevance of carbon dioxide metabolism in Streptococcus thermophilus. Microbiology 155, 1953-1965.

Barbaccia, M.L., 2004. Neurosteroidogenesis: relevance to neurosteroid actions in brain and modulation by psychotropic drugs. Crit. Rev. ${ }^{\mathrm{TM}}$ Neurobiol. 16.

Borovska, J., Vyklicky, V., Stastna, E., Kapras, V., Slavikova, B., Horak, M., Chodounska, H., Vyklicky Jr, L., 2012. Access of inhibitory neurosteroids to the NMDA receptor. Br. J. Pharmacol. 166, 1069-1083.

Bortolato, M., Muroni, A., Marrosu, F., 2007. Treatment of Tourette's syndrome with finasteride. Am. J. Psychiatry 164, 1914.

Bortolato, M., Frau, R., Orru, M., Bourov, Y., Marrosu, F., Mereu, G., Devoto, P., Gessa, G.L., 2008. Antipsychotic-like properties of 5- $\alpha$-reductase inhibitors. Neuropsychopharmacology 33, 3146-3156.

Bortolato, M., Cannas, A., Solla, P., Bini, V., Puligheddu, M., Marrosu, F., 2012. Finasteride attenuates pathological gambling in patients with Parkinson disease. J. Clin. Psychopharmacol. 32, 424-425.

Brustovetsky, T., Pellman, J.J., Yang, X.-F., Khanna, R., Brustovetsky, N., 2014. Collapsin response mediator protein 2 (CRMP2) interacts with $\mathrm{N}$-methyl-D-aspartate
(NMDA) receptor and $\mathrm{Na}+/ \mathrm{Ca} 2+$ exchanger and regulates their functional activity. J. Biol. Chem. 289, 7470-7482.

Cabrera-Vera, T.M., Hernandez, S., Earls, L.R., Medkova, M., Sundgren-Andersson, A.K., Surmeier, D.J., Hamm, H.E., 2004. RGS9-2 modulates D2 dopamine receptor-mediated $\mathrm{Ca} 2+$ channel inhibition in rat striatal cholinergic interneurons. Proc. Natl. Acad. Sci. U.S.A. 101, 16339-16344.

Camacho-Arroyo, I., Villamar-Cruz, O., Gonzalez-Arenas, A., Guerra-Araiza, C., 2002. Participation of the $26 \mathrm{~S}$ proteasome in the regulation of progesterone receptor concentrations in the rat brain. Neuroendocrinology 76, 267-271.

Canti, C., Dolphin, A., 2003. Ca V $\beta$ subunit-mediated up-regulation of Ca V 2.2 currents triggered by D2 dopamine receptor activation. Neuropharmacology $45,814-827$.

Carbone Jr., D.J., Hodges, S., 2003. Medical therapy for benign prostatic hyperplasia: sexual dysfunction and impact on quality of life. Int. J. Impot. Res. 15, 299-306.

Cardinal, R.N., Cheung, T.H., 2005. Nucleus accumbens core lesions retard instrumental learning and performance with delayed reinforcement in the rat. BMC Neurosci. 6,9 .

Caruso, D., Abbiati, F., Giatti, S., Romano, S., Fusco, L., Cavaletti, G., Melcangi, R.C., 2015. Patients treated for male pattern hair with finasteride show, after discontinuation of the drug, altered levels of neuroactive steroids in cerebrospinal fluid and plasma. J. Steroid Biochem. Mol. Biol. 146, 74-79.

Codocedo, J.F., Rodríguez, F.E., Huidobro-Toro, J.P., 2009. Neurosteroids differentially modulate P2X4 ATP-gated channels through non-genomic interactions. J. Neurochem. 110, 734-744

Coetzee, W.A., Amarillo, Y., Chiu, J., Chow, A., Lau, D., McCormack, T., Morena, H., Nadal, M.S., Ozaita, A., Pountney, D., 1999. Molecular diversity of K+ channels. Ann. N.Y. Acad. Sci. 868, 233-255.

Corbit, L.H., Muir, J.L., Balleine, B.W., 2001. The role of the nucleus accumbens in instrumental conditioning: evidence of a functional dissociation between accumbens core and shell. J. Neurosci. 21, 3251-3260.

Desrochers, M., Christou, M., Jefcoate, C., Belzil, A., Anderson, A., 1996. New proteins in the rat CYP2B subfamily: presence in liver microsomes of the constitutive $\mathrm{CYP} 2 \mathrm{~B} 3$ protein and the phenobarbital-inducible protein product of alternatively spliced CYP2B2 mRNA. Biochem. Pharmacol. 52, 1311-1319.

Devoto, P., Frau, R., Bini, V., Pillolla, G., Saba, P., Flore, G., Corona, M., Marrosu, F., Bortolato, M., 2012. Inhibition of $5 \alpha$-reductase in the nucleus accumbens counters sensorimotor gating deficits induced by dopaminergic activation. Psychoneuroendocrinology $37,1630-1645$.

Di Chiara, G., 2002. Nucleus accumbens shell and core dopamine: differential role in behavior and addiction. Behav. Brain Res. 137, 75-114.

Drici, M.D., Burklow, T.R., Haridasse, V., Glazer, R.I., Woosley, R.L., 1996. Sex hormones prolong the QT interval and downregulate potassium channel expression in the rabbit heart. Circulation 94, 1471-1474.

Ekins, S., Vandenbranden, M., Ring, B.J., Gillespie, J.S., Yang, T.J., Gelboin, H.V., Wrighton, S.A., 1998. Further characterization of the expression in liver and catalytic activity of CYP2B6. J. Pharmacol. Exp. Ther. 286, 1253-1259.

Elfverson, M., Linde, A.-M., Le Grevès, P., Zhou, Q., Nyberg, F., Johansson, T., 2008 Neurosteroids allosterically modulate the ion pore of the NMDA receptor consisting of NR1/NR2B but not NR1/NR2A. Biochem. Biophys. Res. Commun. 372, 305-308.

Fernandez, F.R., Morales, E., Rashid, A.J., Dunn, R.J., Turner, R.W., 2003. Inactivation of Kv3. 3 potassium channels in heterologous expression systems. J. Biol. Chem. 278, 40890-40898.

Frau, R., Abbiati, F., Bini, V., Casti, A., Caruso, D., Devoto, P., Bortolato, M., 2015. Targeting neurosteroid synthesis as a therapy for schizophrenia-related alterations induced by early psychosocial stress. Schizophr. Res. 168, 640-648.

Frau, R., Mosher, L.J., Bini, V., Pillolla, G., Pes, R., Saba, P., Fanni, S., Devoto, P., Bortolato, M., 2016. The neurosteroidogenic enzyme $5 \alpha$-reductase modulates the role of D 1 dopamine receptors in rat sensorimotor gating. Psychoneuroendocrinology $63,59-67$

Frye, C.A., Edinger, K.L., Lephart, E.D., Walf, A.A., 2010. 3alpha-androstanediol, but not testosterone, attenuates age-related decrements in cognitive, anxiety, and depressive behavior of male rats. Front. Aging Neurosci. 2, 15.

Fukata, Y., Itoh, T.J., Kimura, T., Ménager, C., Nishimura, T., Shiromizu, T., Watanabe, H., Inagaki, N., Iwamatsu, A., Hotani, H., 2002. CRMP-2 binds to tubulin heterodimers to promote microtubule assembly. Nat. Cell Biol. 4, 583-591.

Gervasini, G., Martinez, C., Agúndez, J., Garcia-Gamito, F., Benitez, J., 2001. Inhibition of cytochrome P450 2C9 activity in vitro by 5-hydroxytryptamine and adrenaline. Pharmacogenet. Genomics 11, 29-37.

Giuliano, F., Allard, J., 2001. Dopamine and sexual function. Int. J. Impot. Res. 13.

González-Flores, O., Guerra-Araiza, C., Cerbón, M., Camacho-Arroyo, I., Etgen, A.M., 2004. The $26 \mathrm{~S}$ proteasome participates in the sequential inhibition of estrous behavior induced by progesterone in rats. Endocrinology 145, 2328-2336.

Grimm, A., Schmitt, K., Lang, U.E., Mensah-Nyagan, A.G., Eckert, A., 2014. Improvement of neuronal bioenergetics by neurosteroids: implications for age-related neurodegenerative disorders. Biochim. Biophys. Acta (BBA)-Mol. Basis Dis. 1842, 2427-2438.

Hatsuzawa, K., Hirose, H., Tani, K., Yamamoto, A., Scheller, R.H., Tagaya, M., 2000 Syntaxin 18, a SNAP receptor that functions in the endoplasmic reticulum, inter- 
mediate compartment, and cis-Golgi vesicle trafficking. J. Biol. Chem. 275, 13713-13720.

Hedblom, E., Kirkness, E.F., 1997. A novel class of GABAA receptor subunit in tissues of the reproductive system. J. Biol. Chem. 272, 15346-15350.

Hensley, K., Venkova, K., Christov, A., Gunning, W., Park, J., 2011. Collapsin response mediator protein-2: an emerging pathologic feature and therapeutic target for neurodisease indications. Mol. Neurobiol. 43, 180-191.

Hesse, L.M., Venkatakrishnan, K., von Moltke, L.L., Duan, S.X., Shader, R.I., Greenblatt, D.J., 2000. CYP2B6 mediates the in vitro hydroxylation of bupropion: potential drug interactions with other antidepressants. Drug Metab. Dispos. 28, 1176-1183.

Ikemoto, S., Panksepp, J., 1999. The role of nucleus accumbens dopamine in motivated behavior: a unifying interpretation with special reference to reward-seeking. Brain Res. Rev. 31, 6-41.

Irwig, M.S., 2012. Depressive symptoms and suicidal thoughts among former users of finasteride with persistent sexual side effects. J. Clin. Psychiatry 73, 1220-1223.

Johansson, T., Frändberg, P.-A., Nyberg, F., Le Grevès, P., 2008. Molecular mechanisms for nanomolar concentrations of neurosteroids at NR1/NR2B receptors. J. Pharmacol. Exp. Ther. 324, 759-768.

Johansson, T., Elfverson, M., Zhou, Q., Nyberg, F., 2010. Allosteric modulation of the NMDA receptor by neurosteroids in rat brain and the impact of long term morphine administration. Biochem. Biophys. Res. Commun. 401, 504-508.

Kaufman, K.D., Olsen, E.A., Whiting, D., Savin, R., DeVillez, R., Bergfeld, W., Price, V.H., Van Neste, D., Roberts, J.L., Hordinsky, M., Shapiro, J., Binkowitz, B., Gormley, G.J., 1998. Finasteride in the treatment of men with androgenetic alopecia: finasteride male pattern hair loss study group. J. Am. Acad. Dermatol. $39,578-589$.

Klinge, C.M., 2008. Estrogenic control of mitochondrial function and biogenesis. J. Cell. Biochem. 105, 1342-1351.

Koethe, D., Bortolato, M., Piomelli, D., Leweke, F., 2008. Improvement of general symptoms in a chronic psychotic patient treated with finasteride: case report. Pharmacopsychiatry $41,115-116$.

Kornberg, A., Lieberman, I., Simms, E.S., 1954. Enzymatic synthesis of pyrimidine and purine nucleotides. 1: I. Formation of 5-phosphoribosylpyrophosphate. J. Am. Chem. Soc. 76, 2027-2028.

Lieberman, I., Kornberg, A., Simms, E.S., 1955. Enzymatic synthesis of pyrimidine nucleotides. Orotidine-5'-phosphate and uridine-5'-phosphate. J. Biol. Chem. 215, 403-415.

McClellan, K.J., Markham, A., 1999. Finasteride: a review of its use in male pattern hair loss. Drugs57, 111-126.

Mi, H., Poudel, S., Muruganujan, A., Casagrande, J.T., Thomas, P.D., 2016. PANTHER version 10: expanded protein families and functions, and analysis tools. Nucleic Acids Res. 44, D336-D342.

Nakajima, K.i., Hirose, H., Taniguchi, M., Kurashina, H., Arasaki, K., Nagahama, M., Tani, K., Yamamoto, A., Tagaya, M., 2004. Involvement of BNIP1 in apoptosis and endoplasmic reticulum membrane fusion. EMBO J. 23, 3216-3226.

Neelands, T.R., Macdonald, R.L., 1999. Incorporation of the $\pi$ subunit into functional $\gamma$-aminobutyric acidA receptors. Mol. Pharmacol. 56, 598-610.

Nudler, S., Pagani, M., Urbano, F., McEnery, M., Uchitel, O., 2005. Testosterone mod ulates $\mathrm{Ca}$ v2. 2 calcium channels' functional expression at rat levator ani neuromuscular junction. Neuroscience 134, 817-826.

Paba, S., Frau, R., Godar, S.C., Devoto, P., Marrosu, F., Bortolato, M., 2011. Steroid $5 \alpha$-reductase as a novel therapeutic target for schizophrenia and other neuropsychiatric disorders. Curr. Pharmaceut. Des. 17, 151-167.

Papadopoulos, V., Baraldi, M., Guilarte, T.R., Knudsen, T.B., Lacapère, J.-J., Lindemann, P., Norenberg, M.D., Nutt, D., Weizman, A., Zhang, M.-R., 2006. Translocator protein $(18 \mathrm{kDa})$ : new nomenclature for the peripheral-type benzodiazepine receptor based on its structure and molecular function. Trends Pharmacol. Sci. 27, 402-409

Paxinos, G., Watson, C., 1998. The Rat Brain in Stereotaxic Coordinates, 6th edition Elsevier/Academic Press, New York.

Piras, C., Soggiu, A., Bonizzi, L., Gaviraghi, A., Deriu, F., De Martino, L., Iovane, G., Amoresano, A., Roncada, P., 2012. Comparative proteomics to evaluate multi drug resistance in Escherichia coli. Mol. Biosyst. 8, 1060-1067.

Piras, C., Soggiu, A., Bonizzi, L., Greco, V., Ricchi, M., Arrigoni, N., Bassols, A., Urbani, A., Roncada, P., 2015. Identification of immunoreactive proteins of Mycobacterium avium subsp paratuberculosis. Proteomics 15, 813-823.

Rae, J.L., Shepard, A.R., 2000. Kv3. 3 potassium channels in lens epithelium and corneal endothelium. Exp. Eye Res. 70, 339-348.
Rahimi-Ardabili, B., Pourandarjani, R., Habibollahi, P., Mualeki, A., 2006. Finasteride induced depression: a prospective study. BMC Clin. Pharmacol. 6, 7.

Ramanathan, S., Tkatch, T., Atherton, J.F., Wilson, C.J., Bevan, M.D., 2008. D2-like dopamine receptors modulate SKCa channel function in subthalamic nucleus neurons through inhibition of Cav2. 2 channels. J. Neurophysiol. 99, 442-459.

Riedl, A.G., Watts, P.M., Douek, D.C., Edwards, R.J., Boobis, A.R., Rose, S., Jenner, P., 2000. Expression and distribution of CYP2C enzymes in rat basal ganglia. Synapse 38, 392-402.

Robertson, C.L., Puskar, A., Hoffman, G.E., Murphy, A.Z., Saraswati, M., Fiskum, G., 2006. Physiologic progesterone reduces mitochondrial dysfunction and hippocampal cell loss after traumatic brain injury in female rats. Exp. Neurol. 197, 235-243.

Roncada, P., Bortolato, M., Frau, R., Saba, P., Flore, G., Soggiu, A., Pisanu, S., Amoresano, A., Carpentieri, A., Devoto, P., 2009. Gating deficits in isolation-reared rats are correlated with alterations in protein expression in nucleus accumbens. J. Neurochem. 108, 611-620.

Ronzitti, G., Bucci, G., Emanuele, M., Leo, D., Sotnikova, T.D., Mus, L.V., Soubrane, C.H., Dallas, M.L., Thalhammer, A., Cingolani, L.A., 2014. Exogenous $\alpha$-synuclein decreases raft partitioning of Cav2. 2 channels inducing dopamine release. J. Neurosci. 34, 10603-10615.

Rudy, B., Chow, A., Lau, D., Amarillo, Y., Ozaita, A., Saganich, M., Moreno, H., Nadal, M.S., Hernandez-Pineda, R., Hernandez-Cruz, A., 1999. Contributions of Kv3 channels to neuronal excitability. Ann. N. Y. Acad. Sci. 868, 304-343.

Sedlacek, M., Korinek, M., Petrovic, M., Cais, O., Adamusova, E., Chodounska, H., Vyklický, L., 2008. Neurosteroid modulation of ionotropic glutamate receptors and excitatory synaptic transmission. Physiol. Res. 57, S49.

Sherif, F.M., 1994. GABA-transaminase in brain and blood platelets: basic and clinical aspects. Prog. Neuro-Psychopharmacol. Biol. Psychiatry 18, 1219-1233.

Soggiu, A., Piras, C., Hussein, H.A., De Canio, M., Gaviraghi, A., Galli, A., Urbani, A., Bonizzi, L., Roncada, P., 2013. Unravelling the bull fertility proteome. Mol. Biosyst. 9, 1188-1195.

Stern, E.A., Kincaid, A.E., Wilson, C.J., 1997. Spontaneous subthreshold membrane potential fluctuations and action potential variability of rat corticostriatal and striatal neurons in vivo. J. Neurophysiol. 77, 1697-1715.

Tillakaratne, N.J., Medina-Kauwe, L., Gibson, K.M., 1995. Gamma-aminobutyric acid (GABA) metabolism in mammalian neural and nonneural tissues. Comp. Biochem. Physiol. Part A: Physiol. 112, 247-263.

Traish, A.M., Hassani, J., Guay, A.T., Zitzmann, M., Hansen, M.L., 2011. Adverse side effects of $5 \alpha$-reductase inhibitors therapy: persistent diminished libido and erectile dysfunction and depression in a subset of patients. J. Sex Med. 8, 872-884.

Traish, A.M., Melcangi, R.C., Bortolato, M., Garcia-Segura, L.M., Zitzmann, M., 2015. Adverse effects of $5 \alpha$-reductase inhibitors: what do we know, don't know, and need to know?. Rev. Endocr. Metab. Disord. 16, 177-198.

Uchimura, N., Cherubini, E., North, R., 1989. Inward rectification in rat nucleus accumbens neurons. J. Neurophysiol. 62, 1280-1286.

Vyklicky, V., Krausova, B., Cerny, J., Balik, A., Zapotocky, M., Novotny, M., Lichnerova, K., Smejkalova, T., Kaniakova, M., Korinek, M., 2015. Block of NMDA receptor channels by endogenous neurosteroids: implications for the agonist induced conformational states of the channel vestibule. Sci. Rep. 5.

Wang, Q., Wang, L., Wardwell-Swanson, J., 1998. Modulation of cloned human neuronal voltage-gated potassium channels (hKv1. 1 and $\mathrm{hKv} 2.1$ ) by neurosteroids. Pflügers Arch. 437, 49-55.

Wilson, C.J., Kawaguchi, Y., 1996. The origins of two-state spontaneous membrane potential fluctuations of neostriatal spiny neurons. J. Neurosci. 16, 2397-2410.

Wolf, J.A., Moyer, J.T., Lazarewicz, M.T., Contreras, D., Benoit-Marand, M., O'Donnell, P., Finkel, L.H., 2005. NMDA/AMPA ratio impacts state transitions and entrainment to oscillations in a computational model of the nucleus accumbens medium spiny projection neuron. J. Neurosci. 25, 9080-9095.

Wong, C.-M., Tsang, S.-Y., Yao, X., Chan, F.L., Huang, Y., 2008. Differential effects of estrogen and progesterone on potassium channels expressed in Xenopus oocytes. Steroids 73, 272-279.

Zhu, A.Z., Cox, L.S., Nollen, N., Faseru, B., Okuyemi, K.S., Ahluwalia, J.S., Benowitz, N.L., Tyndale, R.F., 2012. CYP2B6 and Bupropion's smoking-cessation pharmacology: the role of hydroxybupropion. Clin. Pharmacol. Ther. 92, 771-777.

Zimmer, H.-G., 1998. Significance of the 5-phosphoribosyl-1-pyrophosphate pool for cardiac purine and pyrimidine nucleotide synthesis: studies with ribose, adenine, inosine, and orotic acid in rats. Cardiovasc. Drugs Ther. 12, 179-187.

da Fonseca, P.C., Morris, E.P., 2008. Structure of the Human 26S Proteasome subunit radial displacement open the gate into the proteolytic core. J. Biol. Chem. 283, 23305-23314. 